\title{
Acute myeloid leukaemia presenting as cholestatic hepatitis
}

\section{F A Wandroo, J Murray, D Mutimer, S Hubscher}

J Clin Pathol 2004;57:544-545. doi: 10.1136/jcp.2003.013565

\begin{abstract}
A 40 year old man presented with abdominal pain, jaundice, weight loss, and hepatosplenomegaly. Liver function tests revealed cholestatic jaundice and a computed tomography scan showed an enlarged liver, with a normal biliary tree. Liver biopsy showed diffuse infiltration by neutrophils, monocytoid cells, and blasts. Peripheral blood film and bone marrow were consistent with acute myeloid leukaemia. After treatment with chemotherapy using an acute myeloid leukaemia protocol (UK Medical Research Council AML12), there was complete resolution of jaundice and the patient went into complete molecular remission.
\end{abstract}

$\mathrm{J}$ aundice is rarely a presenting feature of acute myeloid leukaemia. More often, it is treatment related. Obstructive jaundice has been reported on rare occasions in acute myeloid leukaemia (AML). ${ }^{1}$ We report a patient with AML who presented with cholestatic jaundice as a result of hepatic sinusoidal infiltration, which regressed with chemotherapy.

\section{CASE REPORT}

A 40 year old man presented in January 2000 with a history of abdominal pain of one month's duration with some preceding cough, coryzal symptoms, and loss of appetite. He was suffering from longstanding depression and had been on antidepressants (paroxetine) for one year. Physical examination showed pyrexia cachexia, jaundice, few palpable cervical lymph nodes, and hepatosplenomegaly.

Laboratory investigations showed a haemoglobin concentration of $114 \mathrm{~g} /$ litre, a white blood cell count of $23.4 \times 10^{9} /$

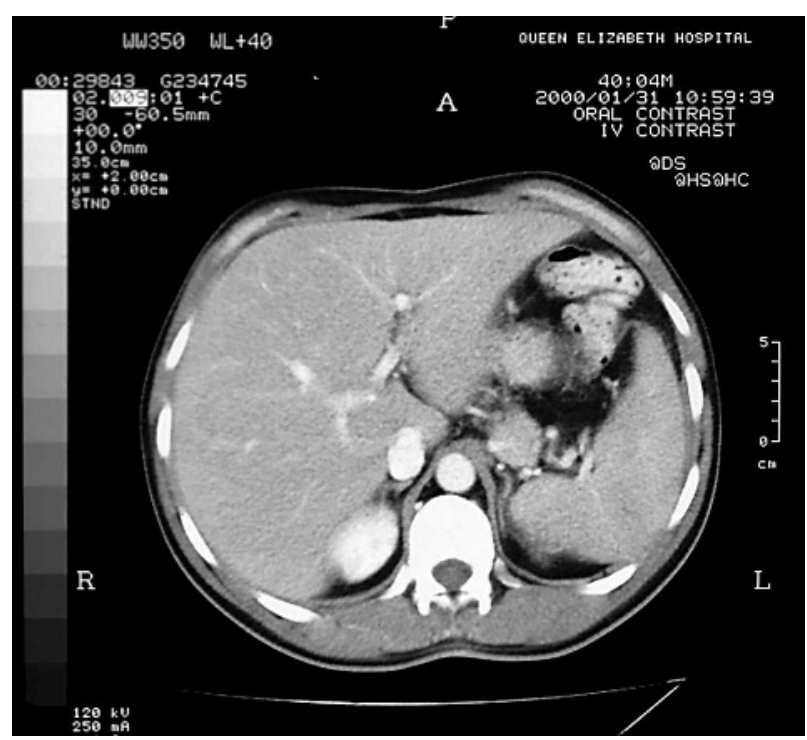

Figure 1 Computed tomography scan of abdomen showing hepatomegaly with normal biliary tree. litre, and a platelet count of $75 \times 10^{9}$ /litre. The differential count showed: neutrophils, $6.3 \times 10^{9} /$ litre; lymphocytes, $3.7 \times 10^{9}$ /litre; monocytes, $12.4 \times 10^{9}$ /litre; myelocytes $0.210^{9}$ /litre; eosinophils, $0.510^{9}$ /litre; and blasts, $0.2 \times 10^{9} /$ litre.

Examination of the blood film showed many dysplastic monocytes with occasional monoblasts. Bone marrow aspirate showed $22 \%$ monocytes, $17 \%$ blasts, $0.7 \%$ promonocytes, and $20 \%$ dysplastic eosinophils. Bone marrow trephine showed an increased number of myeloid and monocytoid cells, with few blasts.

Immunophenotyping of the bone marrow sample showed that $85 \%$ of cells were positive for CD13 and CD33; $10 \%$ of these cells were CD34 positive, $38 \%$ CDllb positive, $46 \%$ CDllc positive, and 29\% CDI4 positive. Cytogenetics on the bone marrow sample showed inversion of chromosome 16 and an additional chromosome 2 (46,XY, add (2)(q37), inv (16) (p13q22)). Molecular analysis of the bone marrow by polymerase chain reaction confirmed the presence of the CBFB-MYHll fusion gene. These results were consistent with the diagnosis of AML with abnormal bone marrow eosinophils (inv (16) (p13q22), CBFB-MYH11) according to the World Health Organisation classification. ${ }^{2}$ Serum bilirubin was $80 \mu \mathrm{mol} /$ litre (normal range, 3-17), alkaline phosphatase was $1258 \mathrm{U} /$ litre (normal range, 30-150), and aspartate aminotransferase was 142 IU/litre (normal range, 5-35). Serological tests for hepatitis viruses were negative. A computed tomography scan showed enlarged liver and spleen with altered echogenicity of the liver but normal biliary ducts (fig 1). Liver biopsy showed diffuse sinusoidal infiltration by a pleomorphic population of cells, mostly consisting of neutrophils and monocytes, with some immature blasts. There was moderate perivenular cholestasis. A similar cellular infiltrate was also present in the portal tracts. Immunohistochemistry showed that most of the cells infiltrating the portal tracts and sinusoids expressed the myeloid marker CD15 and macrophage/monocyte marker CD68, whereas a few sinusoidal cells were positive for CD34, a marker for haemopoeitic precursor cells (fig 2). The patient became progressively unwell with pyrexia, hypoxia, and a rise in bilirubin ( $413 \mu \mathrm{mol} /$ litre), aspartate aminotransferase (172 IU/litre), and alkaline phosphatase (1442 U/litre). He was treated according to the UK Medical Research Council (UK MRC-12) protocol and showed a very good response to chemotherapy (dose adjusted for liver dysfunction), with liver function tests resolving back to normal. He went on to receive four courses of chemotherapy on this protocol and is currently (four years after diagnosis) in complete cytogenetic and molecular remission with normal liver function tests.

\section{DISCUSSION}

Jaundice is rarely seen as a presenting feature in AML. It can occur as a result of drug induced hepatocellular damage, obstruction to the flow of bile, post transfusion viral hepatitis, or infiltration of liver by the leukaemic process or

Abbreviations: $A M L$, acute myeloid leukaemia 


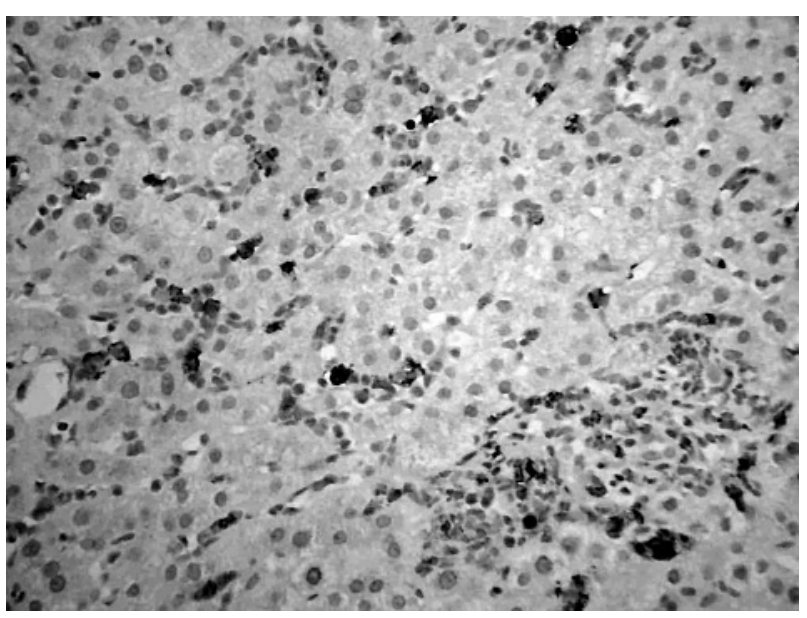

Figure 2 A liver biopsy showing sinusoidal infiltration by CD1 5 positive myeloid cells. A similar infiltrate is present in a small portal tract. Immunoperoxidase staining for CD15.

granulocytic sarcoma. ${ }^{3}$ Most case reports of jaundice in AML are the result of obstructive jaundice secondary to granulocytic sarcoma..$^{3-5}$ Our patient is atypical in that he had interesting hepatic histology with diffuse sinusoidal infiltration of the liver by leukaemic cells. Although our patient was on longterm antidepressant treatment (paroxetine), which can cause liver dysfunction, we confirmed leukaemic infiltration of the liver by liver biopsy. He showed a good response to chemotherapy and went into complete molecular remission, with normal liver function. This case also illustrates that liver dysfunction secondary to leukaemic infiltration can respond to successful treatment (with appropriate dose adjustment of the chemotherapy drugs) of the underlying haematological condition.

\section{Take home messages}

- We report a rare case of acute myeloid leukaemia presenting as jaundice

- Chemotherapy resulted in complete resolution of the jaundice and complete molecular remission

- Thus, liver dysfunction secondary to leukaemic infiltration can respond to successful treatment of the underlying haematological condition

\section{Authors' affiliations}

F A Wandroo, J Murray, D Mutimer, S Hubscher, University of Birmingham Queen Elizabeth Hospital Birmingham, Birmingham B15 $2 S Q$, UK

Correspondence to: FA Wandroo, Department of Haematology, Queen Elizabeth Hospital Birmingham, Birmingham B15 2SQ; fwandroo@ hotmail.com

Accepted for publication 19 November 2003

\section{REFERENCES}

1 Verma N, Prakash D, Marwah N, et al. Granulocytic sarcoma preceding acute myeloid leukaemia. Indian J Pediatr 1989;56:775-6.

2 Haris LH, Jaffe SE, Diebold J, et al. World Health Organisation classification of neoplastic diseases of the haematopoietic and lymphoid tissues: report of the clinical advisory committee meeting-Airlie House, Virginia, November 1997. J Clin Oncol 1999: 17:3835-49.

3 Mclord RG, Gilbert EF, Jod PJ. Acute leukaemia presenting as jaundice with acute liver failure. Clin Pedriatr (Phila) 1973;12:17-20.

4 Abe Y, Takatsuki H, Okada Y, et al. Mucosa-associated lymphoid tissue type lymphoma of the gall bladder associated with acute myeloid leukaemia. Intern Med 1999;38:442-4

5 King DJ, Ewen SWB, Sewell HF, et al. Obstructive jaundice: an unusual presentation of granulocytic sarcoma. Cancer 1987;60:114-17. 